\title{
Carotid Body Tumours: A Review
}

\author{
Abdulsalam Y. Taha \\ Department of Cardiothoracic and Vascular Surgery, School of Medicine, Faculty of Medical Sciences, \\ University of Sulaimaniyah and Sulaimaniyah Teaching Hospital, Sulaimaniyah, Iraq \\ Email: salamyt 1963@hotmail.com
}

Received 24 February 2015; accepted 12 March 2015; published 18 March 2015

Copyright (C) 2015 by author and Scientific Research Publishing Inc. This work is licensed under the Creative Commons Attribution International License (CC BY). http://creativecommons.org/licenses/by/4.0/ (c) (i) Open Access

\begin{abstract}
The carotid body is a small structure weighing $12 \mathrm{mg}$ located in the adventitia of carotid artery bifurcation acting as a chemoreceptor. Carotid body tumour (CBT); formerly known as chemodectoma is a rare, highly vascular, mostly benign tumour arising from the paraganglia of carotid body; hence, the name (carotid paraganglioma). The high vascularity and proximity to cranial nerves and major vessels make this tumour a surgical challenge. Abundant literature has been written about CBT in the last century with a continuous debate regarding its etiology, natural history, biological behavior, proper technique of excision, and the morbidity and mortality associated with its resection. The purpose of this review article is to simplify understanding the basic and clinical aspects of this challenging neoplasm.
\end{abstract}

\section{Keywords}

Carotid Body, Carotid Sinus, Paraganglioma, Neuroendocrine Tumours, Carotid Body Tumours, Review

\section{Introduction}

Carotid body tumours (CBTs) are rare non-chromaffin paragangliomas arising from the chemoreceptor cells found at the carotid bifurcation [1]. The tumour is highly vascular; its blood supply is the richest per gram of tissue of any tumour. Due to its location and high vascularity, this tumour should be diagnosed preoperatively; otherwise, it will face the surgeon with unusual difficulties which make the experience unforgotten. Much literature has been produced about this tumour in the last century with continuing controversy regarding its natural history, biological behavior, proper technique of excision, and the risk of morbidity and mortality [1]-[3].

\section{Carotid Bodies (CB) (Carotid glomus or Glomus caroticum, Latin)}

\subsection{Location}

The carotid bodies, two in number, are situated one on either side of the neck, behind the common carotid artery 
(CCA) within its adventitia at its point of bifurcation into the external (ECA) and internal carotid trunks (ICA) [1].

\subsection{Embryology}

The carotid body originates from the third branchial arch mesoderm and from ectodermal-derived neural crest lineage [4] [5].

\subsection{Structure of Carotid Body}

The first anatomical description of carotid body was provided by Albrecht Von Haller in 1743 [4]. Each CB weights $12 \mathrm{mg}$ and is invested by a fibrous capsule and consists largely of spherical or irregular masses of cells, the masses being more or less separated from one another by septa which extend inward from the deep surface of the capsule [1]. The carotid body is made up of two types of cells, called glomus cells: glomus type I (chief) cells, and glomus type II (sustentacular) cells. Glomus type I/chief cells are derived from the neural crest, which, in turn is derived from the neuroectoderm. Glomus type II/sustentacular cells resemble glia, express the glial marker S100 and act as supporting cells (Figure 1 and Figure 2).

\subsection{Blood and Nerve Supply}

The carotid body is supplied by one or more glomic arteries, which arise from the common or external carotid arteries; and these give rise to a complex mass of small glomic arteries, which have a highly elastic structure, and capillaries [3]. The carotid body contains the most vascularized tissue in the human body. The thyroid gland is very vascular, but not quite as much as the carotid body [1]. There is a profuse afferent nerve supply from the glossopharyngeal nerve to this vascular complex [3].

\section{Carotid Sinus}

It may be that some are not very clear about the distinction between carotid sinus (CS) and carotid body [3]. The sinus is an ill-defined dilatation at the origin of the internal carotid artery in which there is a definite abrupt thinning of the media, which is richly supplied by nerve fibrils [3]. The sinus is concerned with baroreception and

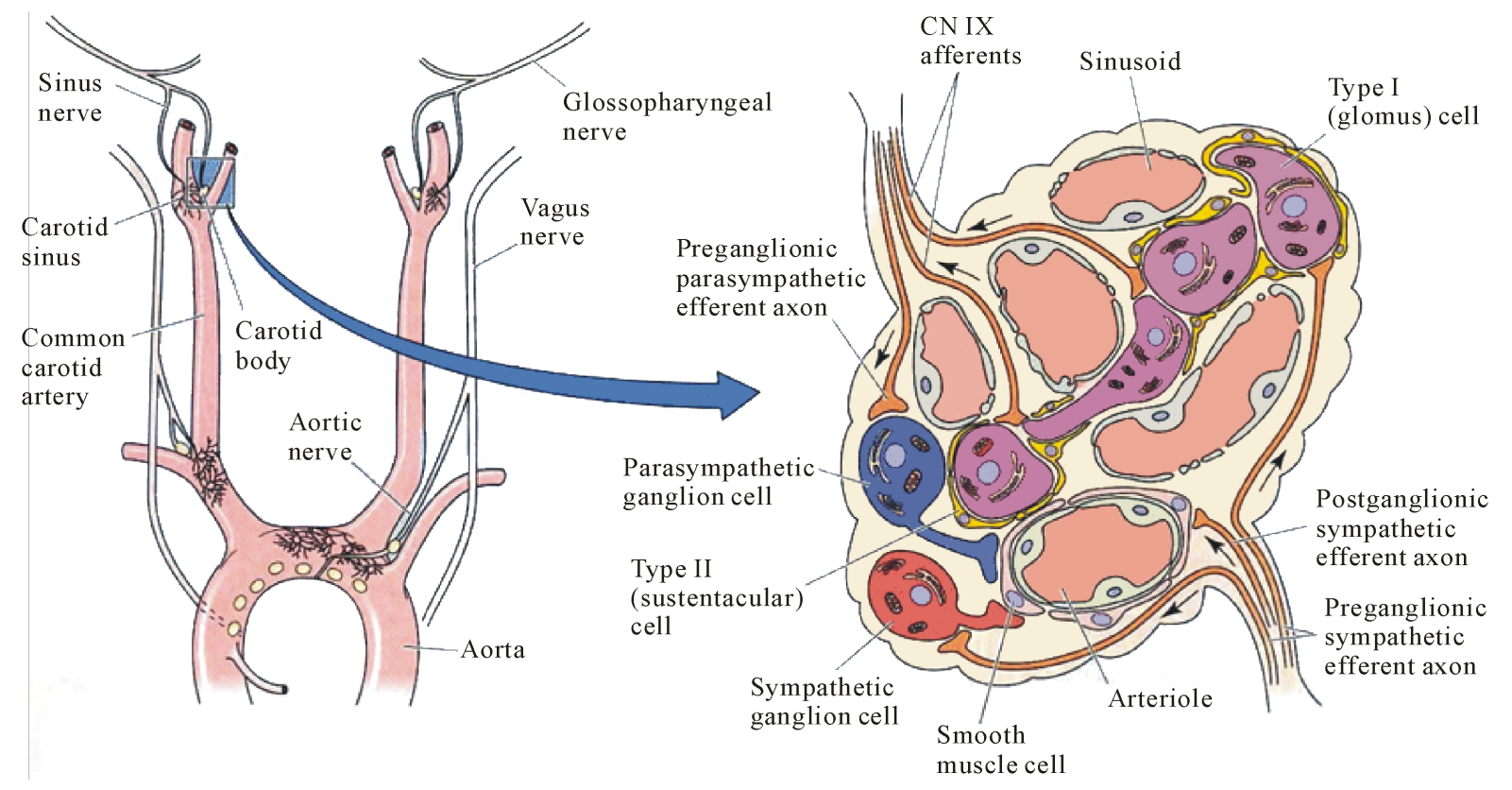

(a)

(b)

Figure 1. Location and microscopic anatomy of carotid body [2]. (a) Location of carotid and aortic bodies; (b) Microscopic anatomy of carotid body. 


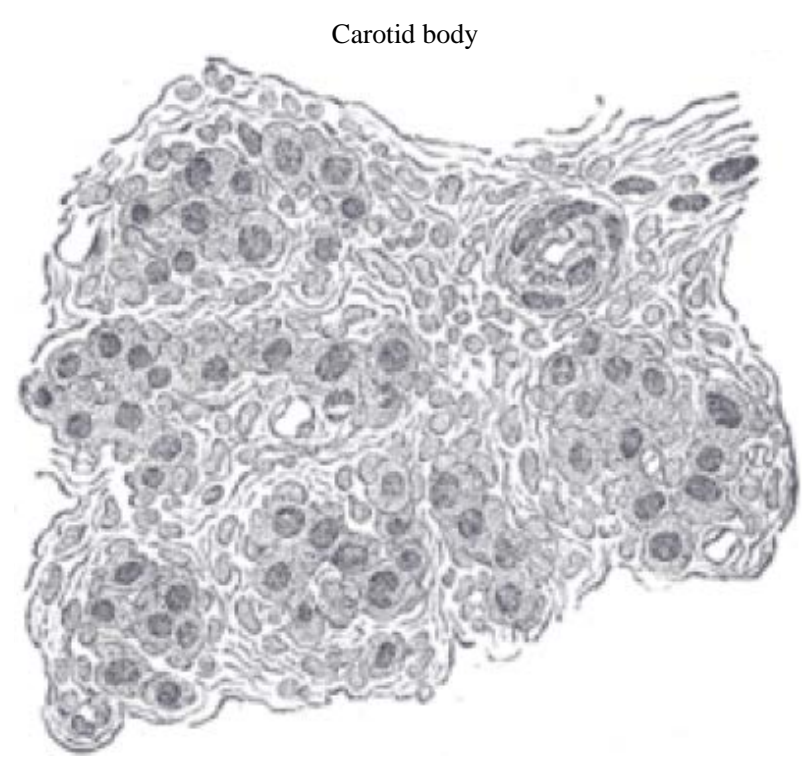

Figure 2. Section of part of human glomus caroticum, highly magnified. Numerous blood vessels are seen in section among the gland cells [1].

physiologists were able to demonstrate in laboratory animals such as the rat how easily and rapidly this thinwalled segment distends and reacts to fluctuations in intravascular pressure [3].

\subsection{The Honorary Thoracic Organs}

This complex arrangement of blood vessels, nerves and gland-like tissue within the carotid bifurcation which comprises the carotid body and sinus was always of interest to all students of thoracic surgery [3]. These structures deserve to be declared as honorary thoracic organs because although they are located in the neck, they appear to be intimately concerned with monitoring vital functions of the heart and lungs such as the maintenance of systemic blood pressure and oxygenation of the blood [3] as we'll see below.

\subsection{Physiology}

The carotid sinus is concerned with baroreception [3] whereas the carotid body acts as a chemoreceptor [3]-[5]. The detailed histological studies of Castro more than 80 years ago led him to a novel interpretation of the role of this mass of nerves and glomus cells around the vascular plexus-namely, that of a chemoreceptor [3]. His interpretation stimulated a series of experiments from distinguished physiologists, who were able to confirm the responsiveness of the carotid body to hypoxia and hypercapnia [3] [4]. Carotid bodies are primarily responsive to hypoxia and to a lesser degree to hypercapnia and acidosis [4] [5] which induce reflex changes in vasomotor activity and respiration [5].

\section{The Neuroendocrine System}

The neuroendocrine system (NES) is made up of the nervous system and the endocrine system. They work together to keep our body functioning regularly. The endocrine glands include the hypothalamus, pituitary, thyroid, parathyroid, adrenal, pancreas, ovaries (in female) and testes (in male). The brain sends some messages to the endocrine system. The endocrine glands release hormones that control important body functions, such as growth, metabolism, reproduction, and response to stress or injury. Hormones help regulate how some of the organs in the body work. Important hormones include serotonin, insulin, adrenaline, estrogen, and testosterone [6].

\subsection{The Neuroendocrine Cells}

The neuroendocrine cells are distributed throughout the body. They release hormones that communicate mes- 
sages to certain cells that are able to receive such messages [6].

\subsection{The Neuroendocrine Tumours}

Like other types of cells in the body, neuroendocrine cells can sometimes develop into cancer. Neuroendocrine tumours (NETs) vary in size and rate of growth, they are generally considered to be malignant. They can metastasize to other parts of the body, most often to the liver or bone [6]. By the time they are diagnosed, more than $50 \%$ of the NETs have already spread to other parts of the body. Some NETs, however, may be found during routine exams and diagnosed at an early stage of the disease [6].

Unlike lung cancer, which arises from the lung, or a breast cancer that arises from the breast, neuroendocrine tumors arise from a cell type. These neuroendocrine tumours can really arise from [almost] anywhere in the body [6].

\subsection{Classification of Neuroendocrine Tumours}

The WHO classified the NETs according to their origin into epithelial and neural:

NETs of epithelial origin:

1) Classical carcinoid.

2) Atypical carcinoid.

3) Small cell neuroendocrine carcinoma (oat cells, intermediate and combined).

NETs of neural origin: paraganglioma [7].

\section{Paragangliomas}

Paragangliomas are rare tumours of neural crest origin [8] (Figure 3). In the head and neck region, paragangliomas most commonly occur as carotid body tumours. Jugulotympanic and vagal paragangliomas are seen less frequently [8]. The paraganglia are located within the paravertebral sympathetic and parasympathetic chains [8], [10] along the pathway of embryologic migration of these paraganglia that extends from the skull base to the

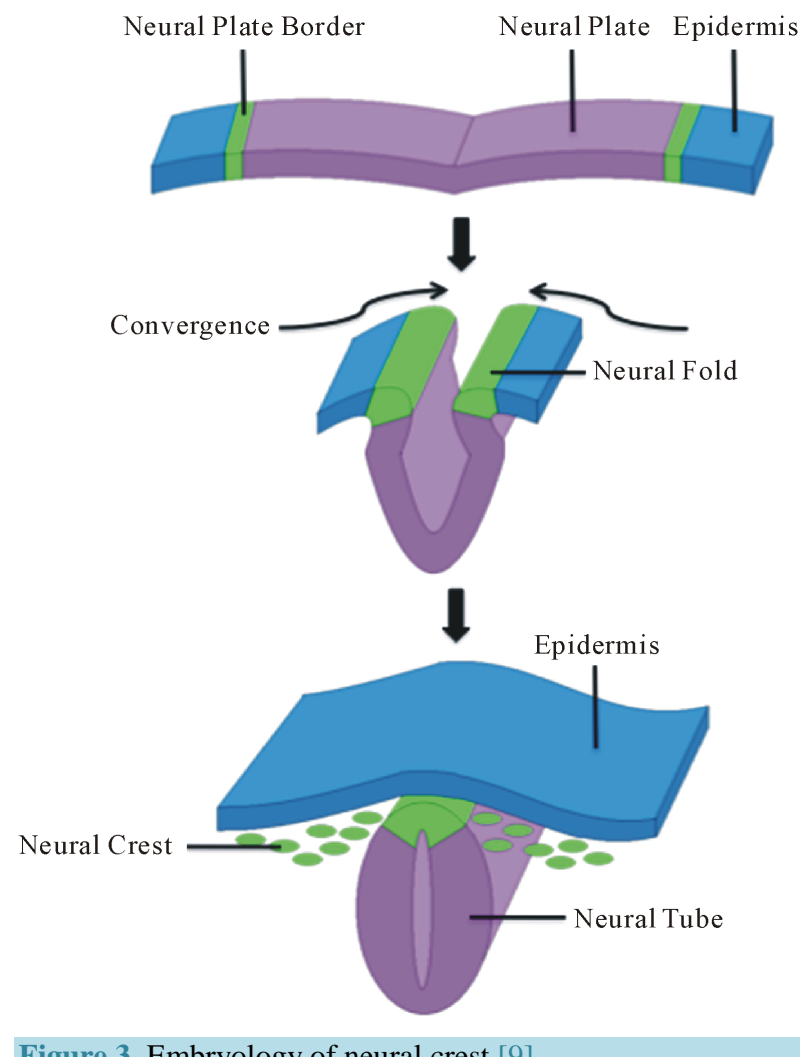

Figure 3. Embryology of neural crest [9]. 
pelvic floor [8]. Common sites of occurrence of paragangliomas include abdomen, retroperitoneum, chest and mediastinum and various head and neck locations such as jugulotympanic membrane, orbit, nasopharynx, larynx, vagal body and carotid body [10]. Paraganglia play an important role in organismic homoeostasis by acting as chemoreceptors or by secretion of catecholamines in response to stress [8]. Carotid body paraganglia are directly connected to the respiratory centers in the central nervous system via the glossopharyngeal nerve [8] whereas the function of other head and neck paraganglia remains unclear [8].

\subsection{Terminology}

Terminology in science and clinical practice regarding tumors of the paraganglial system is divergent [8]. Neumann and coworkers suggested using the term pheochromocytoma for all paragangliomas in the thorax and the abdomen whereas the term head and neck paraganglioma (HNP) is reserved for tumours in the head and neck region [8]. The commonest HNP is carotid body tumour which originates in the paraganglionic cells of the carotid bifurcation [11] while the second commonest type of HNP is a glomus intravagale tumour (GIT), which is derived from closely adjacent paraganglionic tissue located on the vagus nerve [11].

The terms glomus tumour and chemodectoma are often used for HNP [8]. However, strictly speaking, both terms are incorrect and should not be used anymore [8]. The term glomus tumour may be easily mixed up with a benign tumour of the subcutaneous tissue, the so called glomangioma. On the other hand, only the paraganglia of the carotid body act as chemoreceptors; thus, it is incorrect to name all HNPs as chemodectomas [8].

\subsection{Gross Appearance of CBT}

On gross examination the tumors are usually well-circumscribed and may have a pseudocapusle (Figure 4). The cut surface is typically solid with a smooth, rubbery texture but may display some areas of hemorrhage. The actual size of the tumor may vary greatly. Some have reported tumors as large as $10 \mathrm{~cm} \mathrm{[10].}$

\subsection{Histopathology of CBT}

For many decades, pathologists were interested in the characteristic growth pattern of CBT that made a very suitable subject matter for such exercises as the final practical examination of the Royal College of Pathologists [3].

Histologically, carotid body tumors, and paragangliomas in general, have a characteristic growth pattern often referred to as a "zellballen" (Figure 5). This refers to a well-developed nested or organoid growth pattern of the tumor cells with an intervening stromal component of delicate fibrovascular tissue and supporting cells or "sustentacular" cells at the periphery of the zellballen or cell nests. The tumor cells (i.e. paraganglioma cells) are predominantly chief cells with round, hyperchromatic nuclei, a dispersed chromatin and abundant granular cytoplasm which may range from eosinophilic to basophilic in color [10].

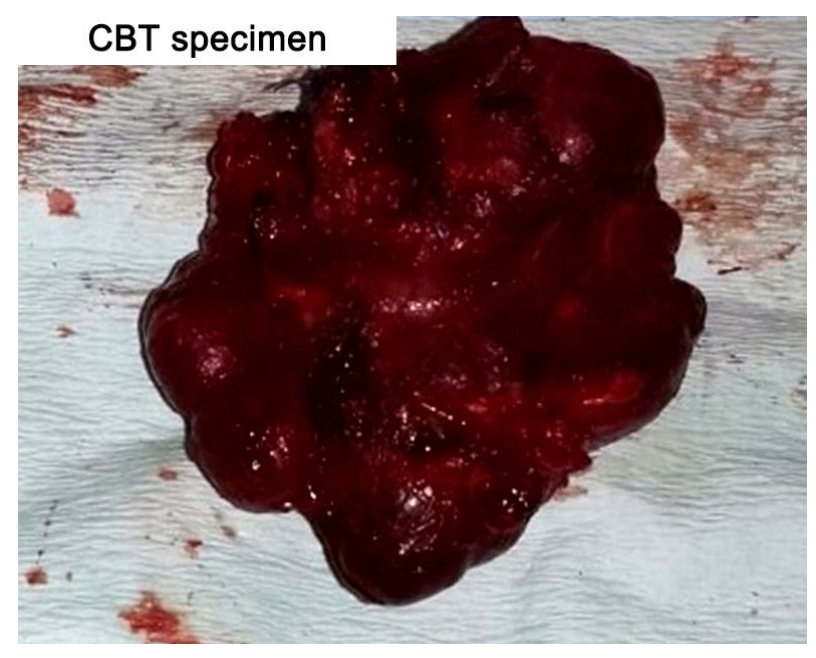

Figure 4. Operative photo of a carotid body tumour. 


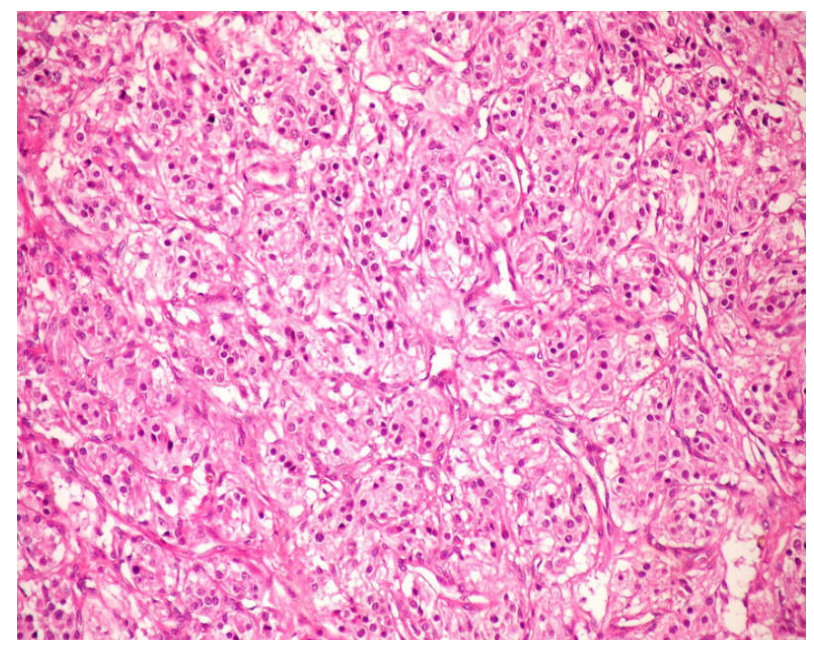

Figure 5. Histopathological appearance of CBT showing a well developed "zellballen" growth pattern. The neoplastic cells demonstrate a basophilic granular cytoplasm and round hyperchromatic nuclei with dispersed chromatin [12] http://www.journalmc.org/index.php/JMC/article/viewFile/17 $\underline{54 / 1018 / 10896}$

The tumor cells show reactivity with chromogranin and synaptophysin stains by immunohistochemical techniques [10] along with other markers of neuroendocrine differentiation such as CD56 and neuron specific enolase [10].

The sustentacular cell population can usually be identified at the periphery of the nests and are thought to be modified Schwann cells; they are spindle shaped and can be highlighted with S-100 protein staining [10].

The ultrastructural character of the carotid body tumour is the neurosecretory granule in the chief cell and the abundance of mitochondria resembling oncocytes of endocrine Glands [5].

Some investigators have suggested that tumours with proliferation of only chief cells are essentially neoplasms, but that tumours composed of both chief and sustentacular cells may be hyperplastic carotid bodies [5].

\subsection{Etiology}

The cause of carotid body tumours is not known [13] [14]. An increased incidence of CBTs in populations living at high altitudes and in patients with chronic obstructive pulmonary disease suggests a role of chronic hypoxia [3]-[8] [10] [11] [13] [14]. It has been suggested to be an extreme degree of hyperplasia associated with high altitude hypoxia, however, no clear explanation for its occurrence at lower elevations has been put forward [10]. Sporadic forms of carotid body tumors are more frequent, while familial forms account for about $10 \%$ of the cases in most series [14]. The tumors are bilateral in 30\% of the familial, but only $5 \%$ of the sporadic cases [13], [14]. The familial form is transmitted in an autosomal dominant pattern [13]. Carotid paragangliomas arising from chronic hypoxia are termed non-heritable [4]. Heritable lesions arise in response to familial genetic characteristics of the multiple endocrine neoplasia type II syndrome, to de novo germ-line mutations in tumour susceptibility genes or occur spontaneously in hyperplastic glands [4]. Approximately $30 \%$ of all HNPs are hereditary and associated with different tumour syndromes [8]. Six genes have been identified and are thought to contribute to the development of pheochromocytoma/paraganglioma [10].

\subsection{Incidence}

Carotid body tumours are very rare neoplasms constituting less than $0.5 \%$ of all body tumours [14]. An incidence of $0.012 \%$ of all surgical specimens has been reported by a hospital-based study describing the paragangliomas of the head and neck region [14]. Approximately 1000 cases had been reported in the literature till 1990s [14]. The Mayo Clinic reported the largest series to-date, describing 153 cases over a period of 50 years [14]. Male and female distribution is equal except at high altitude where females appear to predominate [11]. 


\subsection{Historical Overview}

Mulligan originally described CBTs as chemodectomas [14]. The first attempt at surgical extirpation of carotid paraganglioma was by Reigner in 1880, but the patient died [4] [13]. In 1886, Maydl successfully removed the tumour, leaving the patient aphasic and hemiplegic. The first removal of carotid paraganglioma with preservation of carotid artery complex was by Albert in 1889 [13] and in US by Scudder in 1903 [4] [13]. In 1940 Gordon-Taylor described a safe, sub-adventitial plane of dissection [13] (Figure 6).

In 1957, the mortality and morbidity were so high that Hayes Martin recommended abandonment of resection of difficult tumours on proof of diagnosis [4] [14]. Mortality rate was as high as $10 \%$ till 1970s but they have improved greatly in the current era, largely due to the development of modern imaging and vascular surgical techniques [4] [13]. Recent review of studies shows no mortality. However, perioperative morbidity continues to be high (20\% - 40\%) [4].

\subsection{Natural History}

Carotid body tumours are typically slow growing mass lesions and are often present for years prior to the patient seeking medical attention [10]. This lesion splays apart the ICA and ECA, and as it enlarges, it will encase, but not narrow the arteries [10]. The tumour may attain a large size and infiltrative growth and local recurrence may lead to death [10]. It is important to remember that all CBTs have a malignant potential and it is not always possible to predict malignant behavior based on histological features alone [10]. The large literature on this tumor has indicated that the incidence of malignancy ranges from 2.6 to $50 \%$, and the mortality rate is $8 \%$ in untreated cases. Metastasis, with a ratio considered to be approximately $5 \%$, has been also reported, usually in the regional lymph nodes and uncommonly in the brachial plexus, cerebellum, lungs, bone, abdomen, pancreas, thyroid, kidney and breast [5]. Histopathological analysis is a poor predictor of malignant potential. Malignancy is therefore defined only by the presence of distant metastases. Although 95\% of all CBTs are benign, they remain locally aggressive tumours with growth rates of $2 \mathrm{~cm}$ every five years, which can lead to localized mass effects or neurological dysfunction due to pressure or infiltration [11]. Due to close proximity of the tumour to the lower cranial nerves such as the facial, glossopharyngeal, vagus, accessory, hypoglossal and cervical sympathetic nerves; these nerves can be invaded by the tumour growth and expansion [11].

Some researchers suggest that all CBTs be considered malignant because of their progressive involvement of local neurovascular structures [14]. Although carotid body tumours occur at any age, they are typically diagnosed between the third and sixth decade of life [13]. These tumors are nearly always nonfunctional, but catecholamine-producing tumors do exist and can produce paroxysmal hypertension [13]. No case of spontaneous resolution of these tumors has ever been reported. Rather, carotid body tumors show an inexorable growth pattern. As the tumor grows, the likelihood of CN injury increases [13]. A surgical classification for carotid body tumors was proposed by Shamblin et al in 1971 (Figure 7).

Shamblin group I tumors are relatively small with minimal attachment to the carotid vessels, and surgical excision can be performed without difficulty. Shamblin group II tumors are larger with moderate arterial attachments and can be resected with precise surgical dissection. Group III tumors are large neoplasms encasing the carotid arteries and can be resected only with arterial sacrifice [10] [13].

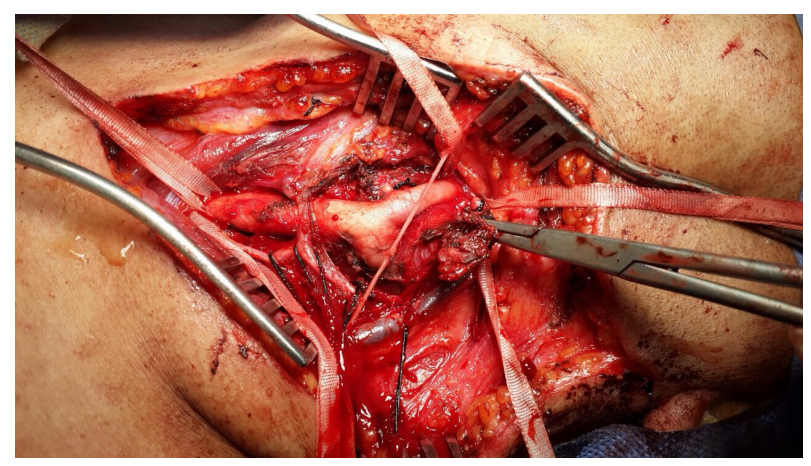

Figure 6. Sub-adventitial dissection for safe resection of CBT. 


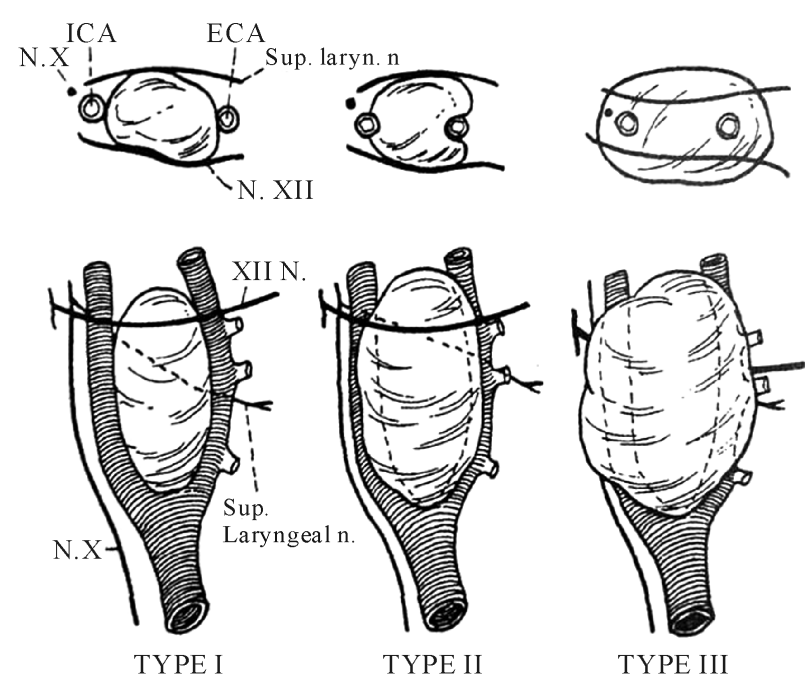

Figure 7. Shamblin classification of CBTs [7].

\subsection{Clinical Features}

Carotid body tumors occur at any age but are typically diagnosed between the third and sixth decades of life [13]. CBTs must be considered in the evaluation of all lateral neck masses [15]. The usual presentation is a slow growing mass at the angle of mandible [4] [14] [15]. Most of carotid body tumors are asymptomatic in the early clinical phase. Eventually, at least $75 \%$ of the patients develop symptoms such as neck pain, neck asymmetry, enlarging neck mass, hoarseness or syncope [11] [13] [14]. Symptoms are generally due to local involvement of nerves and vessels [14] [15]; hoarseness with laryngeal or vagus nerve involvement, dysphagia with glossopharyngeal and hypoglossal nerves involvement [4] [15], Horner's syndrome with invasion or compression of the cervical sympathetic chain, and syncope, which may be due to CS or ICA compression [15]. These tumors are nearly always nonfunctional, but catecholamine-producing tumors do exist and can produce paroxysmal hypertension [4] [11] [13] [14]. Any recent history of uncontrolled hypertension or tachycardia suggests a functional, catecholamine-producing tumor [13]. Functional tumours can secret histamine, serotonin, adrenaline and noradrenaline [11] [14].

On physical examination, CBTs typically present as a non-tender, rubbery, pulsatile mass. Classically, the mass can be displaced laterally but not vertically, due to carotid artery adherence, which is known as a positive Fontaine sign [11] [13]-[15]. Large tumors may cause symptoms from mass effect without constitutional symptoms [13]. An audible bruit (uncommon) [4] [13] [15] or a pulsatile character of the mass suggests further evidence of involvement of or proximity to the carotid bifurcation [15].

Systemic symptoms such as malaise, weight loss, or weakness may suggest metastatic disease [15]. Tachycardia, palpitations, hypertension, and tremors may also indicate possible endocrine activity [15].

Careful neurological examination may reveal deficits of cranial nerves VII, IX, X, XI, or XII [15] as cranial nerve involvement has been estimated as $20 \%$ [4]. Thorough evaluation for primary tumour of the thyroid, the oropharynx, and the nasopharynx is essential, since metastases to a cervical lymph node is a much more frequent cause of a neck mass than paraganglioma [15].

\subsection{Malignant Carotid Paragangliomas}

The estimated incidence of malignancy is between $5 \%$ and $7 \%$. The risk of malignancy is greatest in young patients with heritable tumours. Aggressive local invasion with encirclement of the carotid vessels may suggest the presence of a cancerous lesion; however, documentation of metastasis to regional lymph nodes, liver or lungs is usually required for a carotid paraganglioma to be designated as malignant [4].

\subsection{Functional Carotid Paragangliomas}

All paragangliomas are capable of catecholamine production. About $1 \%$ - 4\% of HNPs can independently se- 
crete sufficient amounts of catecholamine to mimic pheochromocytoma. The pheochromocytoma syndrome is exhibited when catecholamines are produced at 4-5 times the normal. Symptoms of inappropriate catecholamine secretion include labile blood pressure, headaches, cardiac arrhythmias, weight loss, and unusual flushing or sweating [4].

\subsection{Differential Diagnosis}

The differential diagnosis of a non-tender lateral neck mass includes lymphadenopathies, branchial cleft cysts, salivary gland tumors, neurogenic tumors, aneurysms of the carotid artery as well as HNPs [8] [13] [14]. In addition, other neuroendocrine tumors such as medullary thyroid carcinoma and neuroendocrine carcinoma should also be included in the differential diagnosis. Hyalinizing trabecular adenoma of the thyroid gland should also be considered but typically does not display neuroendocrine features on immunohistochemistry [10].

\subsection{Diagnosis}

Carotid body tumors can be a diagnostic challenge for the clinician and lack of pre-operative diagnosis has been reported in up to 30\% of the cases in different series [14]. Despite its rarity, the CBT must be diagnosed preoperatively, since major technical problems can accompany its removal [15]. The patients may be consequently subjected to unwise attempts at biopsy or explorative surgery (Figure 8). Exploration and biopsy can be disastrous and should be avoided in the management of carotid body tumors [4] [14] [15], while fine needle aspiration (FNA) cytology is usually inconclusive [14].

The diagnostic work-up of CBT may involve one or more of the following: Duplex US scanning, CT, MRI, MRA, carotid arteriography, and serum and urinary catecholamine level assessment [4].

\subsubsection{Duplex US Scanning}

In cervical HNPs, B-mode sonography with color-coded Doppler sonography represents an inexpensive, noninvasive diagnostic tool frequently used as the first imaging step. A CBT will typically present as a solid, welldefined, hypoechoic tumor with a splaying of the carotid bifurcation. The external carotid artery is usually displaced anteriorly and medially whereas ICA is typically displaced posteriorly and laterally [8].

\subsubsection{MRI}

Magnetic resonance imaging (MRI) today plays the most important role in the pre-therapeutic evaluation of HNPs. HNPs typically show a hyper-intense signal on T2-weighted images and a distinct contrast enhancement on the T1-weighted images. MRI also presents the exact extension of the tumor and its exact relationship to the carotid vessels [8]. Today, the MRI is performed in combination with a dynamic contrast-enhanced magnetic resonance arteriography (MRA). The MRA helps to diagnose or rule out additional HNPs [8].

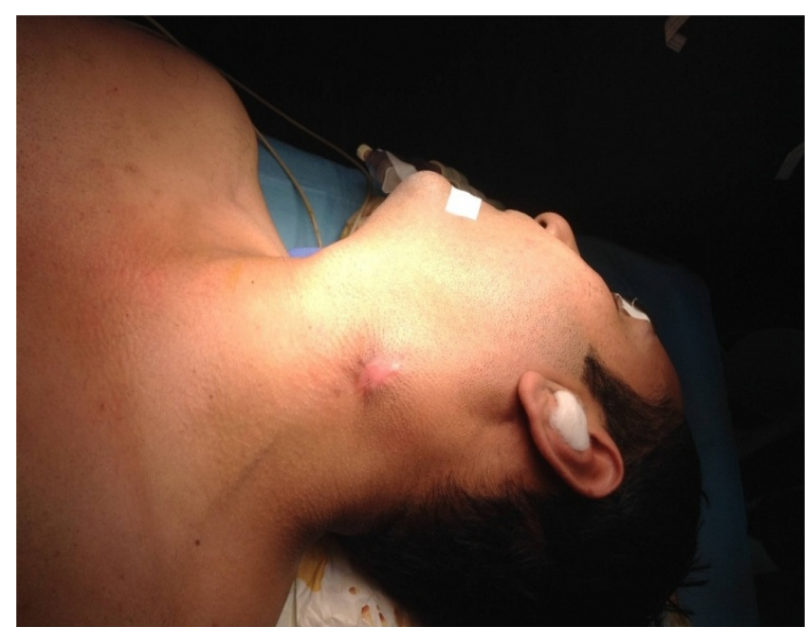

Figure 8. An old scar of previous unsuccessful surgical removal of CBT. 


\subsubsection{Computed Tomography}

Computed tomography (CT) is needed to evaluate jugulotympanic HNPs. The extent of temporal bone destruction is important in the preoperative classification of these tumours and to choose the operative approach accordingly [8]. It is also needed to exclude other paragangliomas in other parts of the body and to detect metastases from advanced CBT (Figure 9).

\subsubsection{Carotid Arteriography}

Carotid arteriography (Figure 10) is the gold standard for diagnosis. It shows the high vascular mass with tumour blush causing splaying of carotid arteries. It should be bilateral to exclude a contralateral tumour. It also reveals the feeder vessels usually from the ECA. Preoperative arteriogram may also include intra-cranial views to determine whether Circle of Willis is intact. Arteriography also affords an opportunity for preoperative embolization of feeder vessels to reduce intra-operative blood loss [4] [8]. It also gives an opportunity for a balloon occlusion test of ICA to determine if the patient would be able to tolerate ligation or sacrifice of the artery should it is necessary [4] [5]. In balloon occlusion test, the ICA is occluded for 15 minutes with electroencephalographic (EEG) monitoring to prove that gross neurologic function remains intact during the procedure [5]. Digital subtraction angiography (DSA) provides an arterial "map" and identifies the blood supply and flow dynamics of the tumor [8]. It is noteworthy that despite its advantages and being the gold standard diagnostic test for CBTs [15], some authors do believe that arteriography should only be performed when there is a need for preoperative tumor embolization [8]. Otherwise, MRA should be used in all other cases due to the invasiveness and potential side effects of arteriography [8].

\subsection{Treatment}

\subsubsection{Surgery}

The treatment modalities for CBTs are surgical excision and/or radiotherapy. Surgical removal is the treatment of choice. Shambling classification is used to assess the difficulty of surgical resection: class I lesions consist of tumours easily isolated and dissected from the carotid vessels (Figure 11); Class II lesions are more adherent to the adventitial layer and partially encircle the vessel at bifurcation; and class III lesions are more densely adherent to the carotid vessels and completely encircle the carotid bifurcation [4].

The routine use of preoperative embolization is controversial because of the potential neurologic complication associated with the accidental reflux of particulate matter into the ophthalmic or cerebral circulation. Some authors advocate its use before the resection of large tumours because it may decrease the vascularity of the tumour, reducing intra-operative blood loss and transfusion requirements. The apparent benefit of embolization should be weighed against the risk of stroke [4]. Its current use is limited to tumours greater than $5 \mathrm{~cm}$ in diameter [4].

The use of shunts during resection of CBTs is controversial. Patetsios et al. believe that familiarity with the

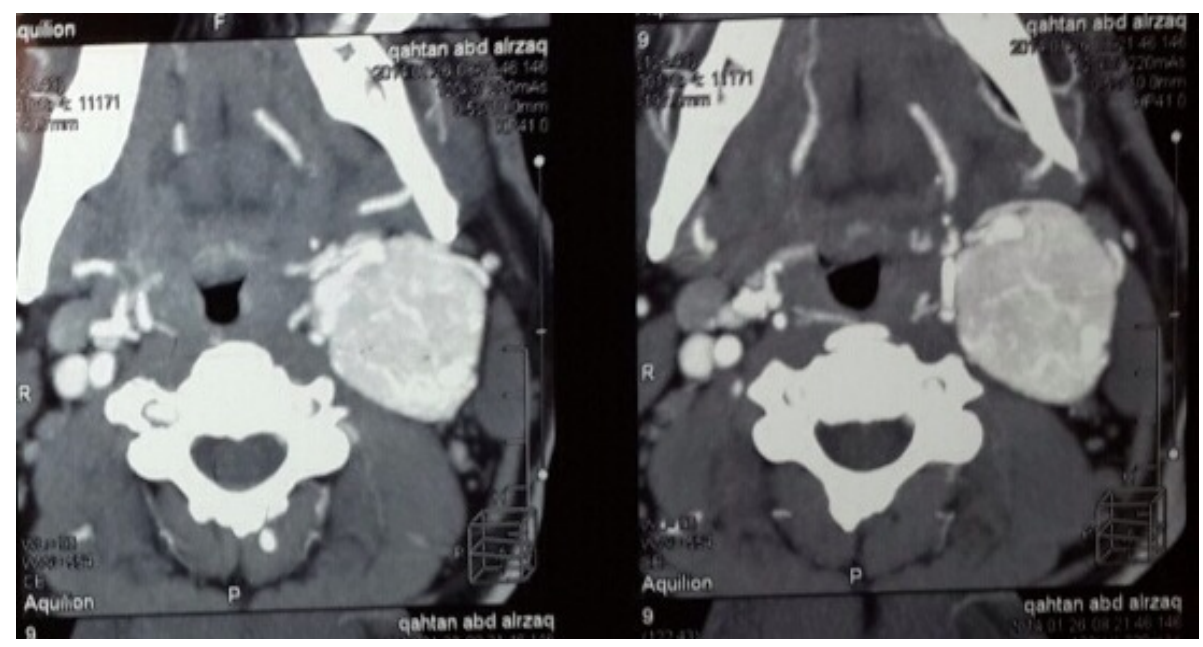

Figure 9. CT scan of the neck showing left CBT. 


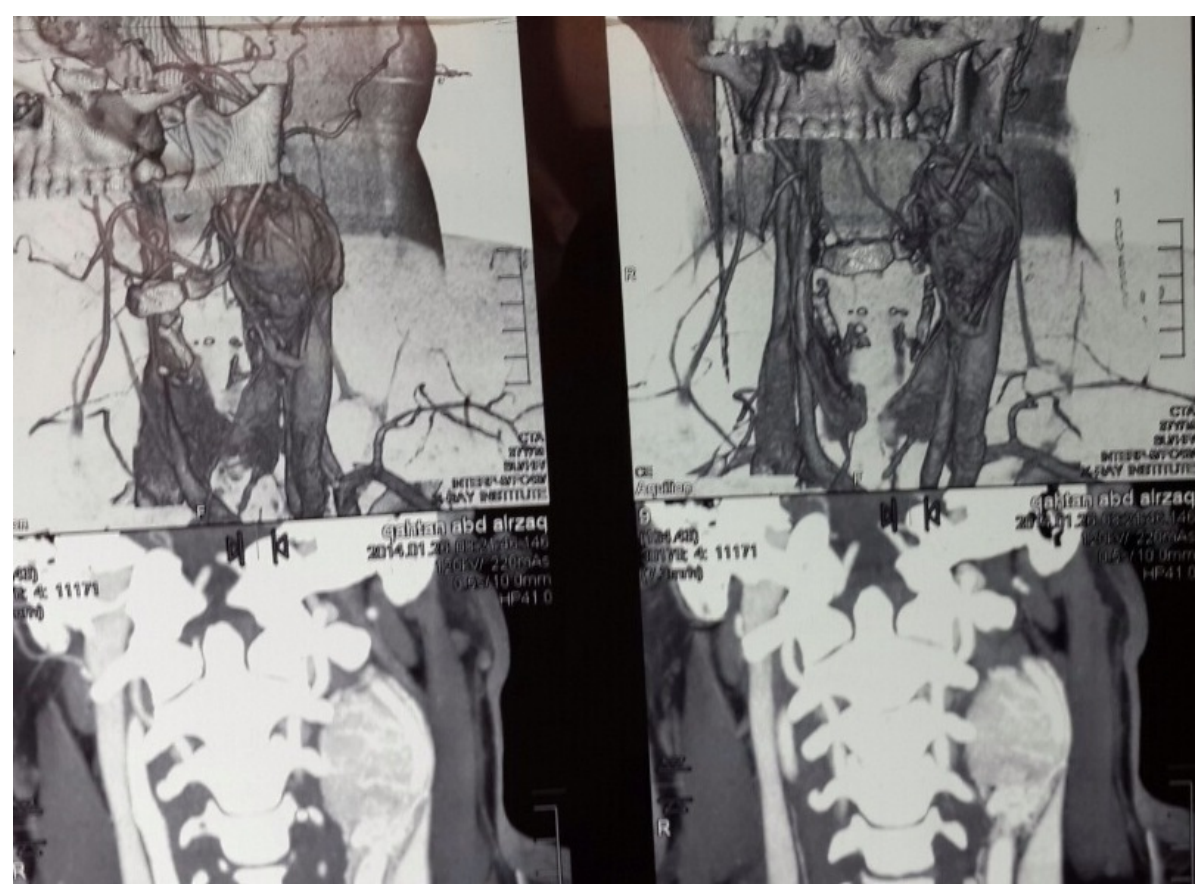

Figure 10. CT angiography of the patient in Figure 9 showing left CBT.

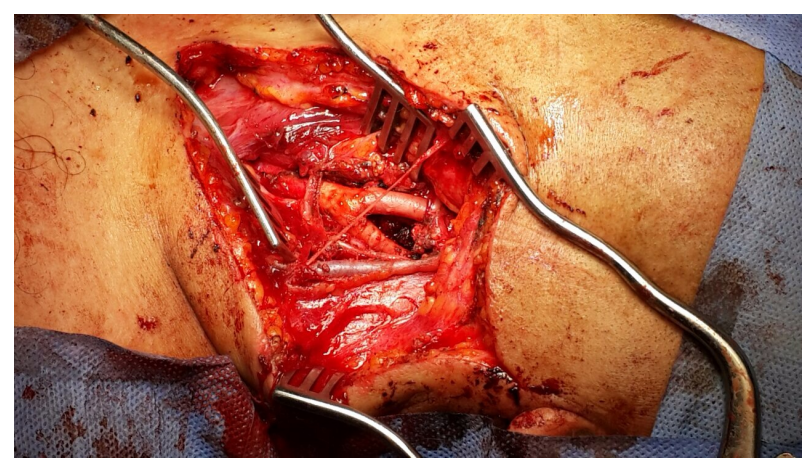

Figure 11. Operative photo after removal of CBT. Figure 4, Figure 6, Figures 8-11 from Prof. Waleed M. Hussen (MBChB. MS., FIBMS. FACS. MRCS. FRCS).

use of intra-luminal vascular shunts is critical since the vessel wall is easily damaged during sub-adventitial dissection [15]. In his institute (Baylor University Medical Center), a temporary in-lying carotid shunt is used routinely whenever vascular reconstruction is required [15]. Moreover, for large tumours, a shunt may be placed through a common carotid arteriotomy before tumour dissection begins to decrease bleeding and prevent an interruption of cerebral blood flow in the event of vessel injury [15]. Previously, they used to utilize temporary shunting only when carotid stump back pressure is $<50 \mathrm{mmHg}$ [15].

Ferreira et al. (2013) from Portugal reported 4 cases of CBTs resected using an ultrasound dissector [16]. They believe that this technique improves the safety of excision, decrease the technical difficulties, lower blood loss and shortens the operation time [16].

\subsubsection{Radiotherapy}

In general, the principal indications for the use of radiotherapy as primary treatment for carotid paragangliomas includes extensive tumours where resection would result in significant morbidity as well as patient related factors such as age and medical condition [4]. Some studies have demonstrated excellent results of radiotherapy as primary treatment for carotid paragangliomas while others believe that radiotherapy is just palliative and best 
reserved for unresectable and multifocal tumours [4] as recurrence has been reported after initial control beside potential complications such as necrosis of the mandible, carotid artery and larynx [4].

\subsubsection{Choice of Therapy}

Controversy is faced everywhere in the literature related to CBT. Treatment options are just an example of this varied opinion. On one hand, surgery is considered by many authors as the standard therapy as it provides an immediate and complete removal of the tumour [4] [17]-[19]. However, the morbidity of cranial nerve injuries and stroke associated with surgery is significant [4] [5] [17]-[20]. On the other hand, the indolent and very slow growth rate of CBT and the fact that most of them are benign and rarely cause death by themselves encourage some authors to adapt the conservative or the so-called (wait and scan) policy [19]. A third group of workers recommend radiotherapy as a primary mode of management of CBTs to achieve a tumour growth control while avoiding the potential morbidity of surgical intervention [19].

\section{Acknowledgements}

We would like to thank Prof. Waleed M. Hussen (MBChB. MS., FIBMS. FACS. MRCS. FRCS) for his permission to use images (Figure 4, Figure 6, Figures 8-11) of his patients in this review article.

\section{Conflict of Interest}

None is declared.

\section{References}

[1] Wikipedia, The Free Encyclopedia (2014) Carotid Body [Internet Monograph]. http://en.wikipedia.org/wiki/Carotid_body

[2] (2014) Location and Microanatomy of Carotid Body Picture [Image on the Internet]. http://163.178.103.176/Fisiologia/respiratorio/pracb_5/Boronfig31-10.jpg

[3] (1983) The Human Carotid Body. Thorax, 38, 561-564. http://dx.doi.org/10.1136/thx.38.8.561

[4] Gupta, B. and Mitra, J.K. (2014) Anaesthetic Management of Chemodectoma Excision. The Indian Anesthetists', Forum [Serial Online]. www.theiaforum.org

[5] Shibuyaa, Y., Umedab, M., Yoshikawab, T., et al. (2002) Carotid Body Tumour: Case Report. Oral Oncology, 38, 313317. http://dx.doi.org/10.1016/S1368-8375(01)00057-4

[6] (2013) Learning about the Neuroendocrine System: Fact Sheet [Internet Monograph]. Basel: The Net Alliance: A Novartis Oncology Initiative. http://www.thenetalliance.com/About-NET.jsp?id=0

[7] Buckingham, E. and Ryan, M. (2002) Paragangliomas [Unpublished Lecture Notes]. The University of Texas Medical Branch, Department of Otolaryngology.

[8] Boedeker, C.C. (2011) Paragangliomas and Paraganglioma Syndromes. GMS Current Topics in Otorhinolaryngology-Head and Neck Surgery [Serial Online]. http://www.egms.de/static/en/journals/cto/2012-10/cto000076.shtml

[9] (2014) Neural Crest Image [Image on the Internet]. http://upload.wikimedia.org/wikipedia/commons/thumb/6/60/Neural_crest.svg/2000px-Neural_crest.svg.png

[10] Wieneke, J.A. and Smith, A. (2009) Paraganglioma: Carotid Body Tumor. Head and Neck Pathology, 3, 303-306. http://dx.doi.org/10.1007/s12105-009-0130-5

[11] O’Neill, S., O’Donnell, M., Harkin, D., Loughrey, M., Lee, B. and Blair, P. (2011) A 22-Year Northern Irish Experience of Carotid Body Tumours. Ulster Medical Journal, 80, 133-140.

[12] Histopathological Appearance of Carotid Body Tumour [Image on the Internet]. http://www.journalmc.org/index.php/JMC/article/viewFile/1754/1018/10896

[13] Wang, S.J., Wang, M.B., Barauskas, T.M. and Calcaterra, T.C. (2000) Surgical Management of Carotid Body Tumors. Otolaryngology — Head and Neck Surgery, 123, 202-206. http://dx.doi.org/10.1067/mhn.2000.106709

[14] Tayyab, M., Khan, M.R. and Sophie, Z. (2003) Presentation and Management of Carotid Body Tumors. Journal of Pakistan Medical Association, (Serial Online), 53. http://www.jpma.org.pk/full_article_text.php?article_id=227

[15] Patetsios, P., Gable, D.R., Garrett, W.V., Lamont, J.P., Kuhn, J.A., Shutze, W.P., et al. (2002) Management of Carotid Body Paragangliomas and Review of a 30-Year Experience. Annals of Vascular Surgery, 16, 331-338.

http://dx.doi.org/10.1007/s10016-001-0106-8 
[16] Ferreira, J., Canedo, A., Braga, S., Vasconcelos, J., Gouveia, R., Martins, V., Brandão, P. and Vaz, A. (2013) Carotid body Tumours Resection with Ultrasound Dissector. Chinese Medical Journal, 126, 586-588.

[17] Hussen, W.M. (2008) Carotid Body Tumor. Journal of Faculty of Medicine Baghdad, 50, 410-413.

[18] Salehian, M.T., Ghoddoosi, I., Marashi, A. and Fazel, I. (2002) Carotid Body Tumours: Survey of 97 Patients. Archives of Internal Medicine, 5, 1-5.

[19] Suarez, C., Rodrigo, J.P., Mendenhall, W.M., Hamoir, M., Silver, C.E., Grégoire, V., Strojan, P., Neumann, H.P., Obholzer, R., Offergeld, C., Langendijk, J.A., Rinaldo, A. and Ferlito, A. (2013) Carotid Body Paragangliomas: A Systematic Study on Management with Surgery and Radiotherapy. European Archives of Oto-Rhino-Laryngology, (Serial Online), 271, 23-34. http://link.springer.com/article/10.1007/s00405-013-2384-5

[20] Kafie, F.E. and Freischlag, J.A. (2001) Carotid Body Tumors: The Role of Preoperative Embolization. Annals of Vascular Surgery, 15, 237-242. http://dx.doi.org/10.1007/s100160010058 